\title{
BMJ Open Risk of disseminated intravascular coagulation in patients with type 2 diabetes mellitus: retrospective cohort study
}

\author{
Kenichiro Nogami, ${ }^{1,2}$ Isao Muraki, ${ }^{1,3}$ Hironori Imano, ${ }^{1}$ Hiroyasu Iso ${ }^{1}$
}

To cite: Nogami K, Muraki I, Imano $\mathrm{H}$, et al. Risk of disseminated intravascular coagulation in patients with type 2 diabetes mellitus: retrospective cohort study. BMJ Open 2017;7:e013894. doi:10.1136/bmjopen-2016013894

- Prepublication history for this paper is available online. To view these files please visit the journal online (http://dx.doi.org/10.1136/ bmjopen-2016-013894).

Received 15 August 2016 Revised 21 December 2016 Accepted 3 January 2017

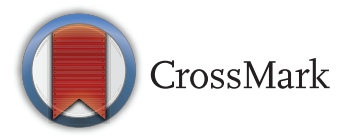

${ }^{1}$ Public Health, Department of Social Medicine, Osaka University Graduate School of Medicine, Osaka, Japan

${ }^{2}$ Takeda Development Center Japan, Takeda

Pharmaceutical Company

Limited, Osaka, Japan

${ }^{3}$ Osaka Center for Cancer and

Cardiovascular Disease

Prevention, Osaka, Japan

Correspondence to

Kenichiro Nogami;

kenichiro_nogami@yahoo.

co.jp

\section{ABSTRACT}

Objectives: To determine quantitatively the association between type 2 diabetes mellitus (T2DM) and disseminated intravascular coagulation (DIC).

Design: Retrospective cohort study using a claims database.

Setting: Medical care institutions representing $9 \%$ of all secondary hospitals (acute care hospitals) in Japan. Participants: In total, 797324 admissions, comprising 435354 patients aged 18-79 years at the time of admission, were enrolled between January 2010 and September 2014. All patients were diagnosed with diabetes or admitted to hospitals that provided laboratory data.

Main outcome measures: Incidence of DIC reported by physicians in claims data.

Results: Logistic regression analysis found that the risk of DIC was significantly higher in T2DM patients than in non-DM patients (fully adjusted OR: 1.39 (95\% Cl 1.32 to 1.45)), independent of age, sex, admission year and potential underlying diseases. The higher risk of DIC in T2DM patients was apparent in those who were treated with insulin within the 30-day period prior to admission (1.53 (1.37 to 1.72$)$ ). When stratified by the potential underlying diseases, the risk of DIC was higher in T2DM patients with non-septic severe infection (1.67 (1.41 to 1.97)) and with solid tumour (1.59 (1.47 to 1.72)) than in non-DM patients with those underlying diseases. The risk was similar between T2DM and non-DM patients with sepsis $(0.98$ (0.90 to 1.08)) and lower in T2DM patients with acute leukaemia (0.70 (0.59 to 0.84$)$ ).

Conclusions: T2DM was associated with a higher risk of DIC, particularly when recently treated with insulin, as well as among admissions with solid tumour or non-septic severe infection.

\section{INTRODUCTION}

Disseminated intravascular coagulation (DIC) is a serious condition characterised by the widespread and persistent activation of coagulation. This results in diffuse microthrombi and consequent organ failure.

\section{Strengths and limitations of this study}

- To the best of our knowledge, this retrospective cohort study using a claims database is the first study quantitatively determining the association between type 2 diabetes mellitus and disseminated intravascular coagulation (DIC).

- The strengths of the present study include large sample size and the use of a nationwide claims database which minimised sampling bias and the possibility of type II error.

- The robustness of the findings of the primary and sensitivity analyses suggest that the probability of type I error in these findings is small.

- The possible misdiagnosis of DIC and limited availability of data before the admission are the major limitations of this study.

Coagulation is followed by fibrinolysis of varying degrees, depending on the underlying disease. Owing to ongoing activation of the coagulation system, haemostatic factors such as platelets and coagulant factors are depleted. This may in turn lead to consumption coagulopathy with bleeding from various sites. The three most common clinical conditions associated with DIC are sepsis, acute leukaemia and solid cancers. ${ }^{1-4}$ According to a nationwide epidemiological survey conducted in Japan, the frequency of DIC was $1.9 \%$ of admissions to medical universities. The rate of fatality was $56.0 \%$, and the annual occurrence of DIC was estimated at 73000 in Japan. ${ }^{5}$ DIC has been shown to be an independent and strong predictor of mortality in patients with sepsis. ${ }^{6} 7$ Relevant diagnosis and treatment in the acute phase are thus important for improving the poor prognosis of this disease.

Diabetes mellitus (hereafter, diabetes) is characterised by fasting hyperglycaemia and is an established risk factor of atherothrombotic disorders. These affect the coronary, cerebral and peripheral arterial trees. 


\section{Study population A}

Study population A is formed excluding the following admissions from eligible population: (1) admission of patients with type 1 diabetes; (2) admission of T2DM patients without any record of previous antidiabetic medication use (to minimise the impact of overdiagnosis and misclassification of T2DM); (3) admission of patients with a history of DIC; (4) admission due to liver failure, liver cirrhosis, vitamin $\mathrm{K}$ deficiency and hypersplenism (to differentiate DIC from these diseases, which can exhibit similar symptoms and laboratory findings) and (5) admission with obstetric complications (because examination of DIC that develops with underlying obstetric complications was outside the scope of the current study). A total of 566191 admissions were included in the study population A (260 091 admissions for T2DM, and 306100 admissions for non-DM).

\section{Study population B}

We first examined the association between T2DM and risk of DIC based solely on the diagnosis in the health insurance claims, because of the limited availability of laboratory data. To evaluate the robustness of these primary findings, we also added the criteria of laboratory data for the diagnosis of DIC in the study population $\mathrm{B}$ restricting to the admissions in the hospitals which provided laboratory data.

\section{Study population C}

To evaluate the effect of antidiabetic medication on the development of DIC, we selected admissions from study population A which had T2DM and had used antidiabetic medication within the 30-day period prior to admission. These admissions formed study population $\mathrm{C}$.

\section{Definition of T2DM and DIC}

In the current study, we defined T2DM as patients with any records of previous antidiabetic medication use in addition to past or present physician's diagnosis of T2DM (ICD-10 code E11-14) in the claim data.

The DIC was defined by the physician's diagnosis of DIC (ICD-10 code D65) based on clinical symptoms and related laboratory findings in study population A and C, but the data for any clinical symptoms and most of laboratory findings was not available in the current database. In study population B, we defined DIC as when patients met the following three criteria during a period of hospitalisation: (1) reported diagnosis of DIC and laboratory findings of (2) platelet counts $<80 \times 10^{9} / \mathrm{L}$ and (3) international normalised ratio of prothrombin time (PT/INR) $\geq 1.2$ on the same day. Owing to the unavailability of laboratory data, we used PT/INR instead of prothrombin ratio (PR) as the criteria of DIC in study population $\mathrm{B}$, although the diagnostic criteria for acutephase DIC established by the Japanese Association for Acute Medicine are platelet counts, fibrin/fibrinogen degradation product (FDP) levels, PR and the presence of inflammatory clinical symptoms (abnormal body temperature, tachycardia, hyperventilation and abnormality of white cell count). We considered the misclassification of DIC non-differential between T2DM and non-T2DM patients, because diabetes is a non-major risk factor for DIC. As a result, any misclassification in this study would more likely dilute rather than skew the true association.

\section{Statistical analysis}

All patients had one or more admissions in the study period, and all comparisons were performed on an admission (not patient) basis. Mean age and proportion of sex, comorbidity and admission year were compared between T2DM and non-DM patients, tested for differences between mean values using Student's t-tests, and for proportions using $\chi^{2}$ tests. We estimated ORs and 95\% CIs for the development of DIC using a logistic regression analysis. Primary adjustment variables were age (continuous), sex (male or female) and admission year ( $\leq 2010,2011,2012,2013$ or 2014).

To examine whether T2DM was directly or indirectly associated with DIC risk, we additionally adjusted for potential underlying diseases that cause DIC, such as sepsis, non-septic severe infection, solid tumour and acute leukaemia (yes or no, for each). To examine the potential interaction between T2DM and underlying conditions in relation to the development of DIC, we stratified analyses by the presence of sepsis, non-septic severe infection, solid tumour or acute leukaemia. To investigate the effect modification by the antidiabetic medications on the development of DIC, we assessed the associations stratified by antidiabetic medications given within the 30-day period prior to admission in study population $\mathrm{C}$.

For all statistical tests, we used a two-tailed significance level of 0.05 and conducted analyses in SAS 9.3 (SAS Institute, Cary, North Carolina, USA).

\section{RESULTS}

DIC was diagnosed in $1.4 \%(7927 / 566191)$ of admissions in study population A $(1.1 \%(3287 / 306100)$ of non-DM patients and 1.8\% (4640/260 091) of T2DM patients), and diagnosis of DIC with supporting laboratory data was seen in $0.4 \%(1128 / 313426)$ of admissions in study population B $(0.3 \%(821 / 259633)$ and $0.6 \%$ (307/53 793), respectively) (figure 1).

Of the 7927 admissions with DIC in study population A, DIC was diagnosed on admission in $75 \%$ and after the date of hospitalisation in $25 \%$. The average period of hospitalisation was $59.0 \pm 68.8$ days, and the outcome was fatal in $32.0 \%$ of admissions. In study population A, the average admission period was $17.4 \pm 27.9$ days, and the outcome was fatal in $3.4 \%$ of admissions without DIC. The DIC was treated by anticoagulant treatment, replacement therapy (platelet concentrates, fresh frozen plasma) or antifibrinolytic treatment in $84.8 \%, 42.3 \%$, and $18.8 \%$ of admissions, respectively. At least one of these therapies was given to $88.1 \%$ of admissions. 
In study population $\mathrm{B}$, the timing of diagnosis of DIC with supporting laboratory data was almost the same as for study population A: diagnosis occurred on admission in $76 \%$. The average period of hospitalisation of admissions with DIC and supporting laboratory data was longer ( $68.5 \pm 80.5$ days) and fatal outcomes were more common $(53.7 \%(606 / 1128))$ than in study population A. At least one of the three treatments for DIC described above was administered in $95.3 \%$ of admissions. The diagnosis of DIC (with and without supporting laboratory data) was made in $0.9 \%$ (2961/ 313426 ) of admissions, and fatal outcome was observed in $36.3 \%(1074 / 2961)$ of the admissions in the study population B.

Compared with non-DM patients, T2DM patients were older (mean \pm SD: $66.9 \pm 9.7$ vs $60.3 \pm 15.2$ years in study population A; $66.7 \pm 9.8$ vs $59.4 \pm 15.6$ years in study population B) and more likely to be men $(67.7 \%$ vs $54.6 \%$ in study population A; $67.9 \%$ vs $53.0 \%$ in study population B) (table 1).

T2DM was associated with more prevalent comorbidity in most of the organ systems. This was particularly the case for complications that had resulted from the diabetes, including diseases of the cardiovascular, renal, ocular and nervous systems. With respect to potential diseases underlying DIC, T2DM patients were either equally or more likely to have sepsis, non-septic serious infections and acute leukaemia than non-DM patients, while solid tumour was more prevalent in non-DM patients.
The age, sex and admission year-adjusted OR for the incidence of DIC in T2DM patients was 1.60 (95\% CI 1.52 to 1.67 ; table 2 ).

After further adjustment based on the diseases underlying DIC, the association was attenuated but remained statistically significant. The fully adjusted OR was 1.39 (1.32 to 1.45). We stratified analyses by the presence of sepsis, non-septic severe infection, solid tumour and acute leukaemia. Compared with non-DM patients, the risk of DIC was higher in admissions with non-septic severe infection (fully adjusted OR: 1.67 (1.41 to 1.97)) or solid tumour (1.59 (1.47 to 1.72)). The risk of DIC was unchanged in admissions with sepsis $(0.98$ (0.90 to $1.08))$, lower in admissions with acute leukaemia $(0.70$ $(0.59$ to 0.84$))$ and even lower in admissions with acute promyelocytic leukaemia (APL) (0.36 (0.16 to 0.82)). In study population $\mathrm{B}$, for which laboratory data were available, we used the reported diagnosis of DIC with supporting laboratory data as the outcome, instead of only the reported diagnosis. These analyses showed that the association between T2DM and the development of DIC was quite similar to the results from the primary analyses.

We calculated case fatality rate of admissions with DIC for total population and according to underlying diseases in study population A (table 3).

The overall case fatality rate in T2DM group is 1.18 times higher than that in non-DM group. When stratified by underlying disease of DIC, the fatality rate in T2DM group is 1.09 times for patients with sepsis, 1.23

Table 1 Baseline characteristics of each study population (non-DM vs T2DM)

\begin{tabular}{|c|c|c|c|c|c|c|}
\hline & \multicolumn{3}{|c|}{$\begin{array}{l}\text { Study population A (overall } \\
\text { population) }\end{array}$} & \multicolumn{3}{|c|}{$\begin{array}{l}\text { Study population B (laboratory } \\
\text { data-available admissions only) }\end{array}$} \\
\hline & non-DM & T2DM & p Value* & non-DM & T2DM & p Value ${ }^{*}$ \\
\hline Number of admission & 306100 & 260091 & & 259633 & 53793 & \\
\hline Male, \% & $54.6 \%$ & $67.7 \%$ & $<0.0001$ & $53.0 \%$ & $67.9 \%$ & $<0.0001$ \\
\hline Age, years (mean $\pm S D)$ & $60.3 \pm 15.2$ & $66.9 \pm 9.7$ & $<0.0001$ & $59.4 \pm 15.6$ & $66.7 \pm 9.8$ & $<0.0001$ \\
\hline \multicolumn{7}{|l|}{ Admission year } \\
\hline 2010 & $14.1 \%$ & $10.9 \%$ & $<0.0001$ & $13.2 \%$ & $10.7 \%$ & $<0.0001$ \\
\hline 2011 & $19.3 \%$ & $16.3 \%$ & & $18.8 \%$ & $18.0 \%$ & \\
\hline 2012 & $22.8 \%$ & $20.9 \%$ & & $22.5 \%$ & $23.2 \%$ & \\
\hline 2013 & $26.0 \%$ & $29.9 \%$ & & $26.2 \%$ & $28.3 \%$ & \\
\hline 2014 & $17.7 \%$ & $22.0 \%$ & & $19.2 \%$ & $19.9 \%$ & \\
\hline \multicolumn{7}{|l|}{ Concomitant diseases } \\
\hline Neural & $28.7 \%$ & $39.3 \%$ & $<0.0001$ & $28.3 \%$ & $39.1 \%$ & $<0.0001$ \\
\hline Eye & $8.1 \%$ & $24.0 \%$ & $<0.0001$ & $7.8 \%$ & $24.0 \%$ & $<0.0001$ \\
\hline Cardiovascular & $45.9 \%$ & $78.6 \%$ & $<0.0001$ & $43.5 \%$ & $78.3 \%$ & $<0.0001$ \\
\hline Respiratory & $25.6 \%$ & $34.3 \%$ & $<0.0001$ & $24.8 \%$ & $33.1 \%$ & $<0.0001$ \\
\hline Gastrointestinal & $63.7 \%$ & $75.4 \%$ & $<0.0001$ & $62.3 \%$ & $75.4 \%$ & $<0.0001$ \\
\hline Renal & $22.6 \%$ & $32.8 \%$ & $<0.0001$ & $22.1 \%$ & $32.5 \%$ & $<0.0001$ \\
\hline Sepsis & $1.6 \%$ & $3.5 \%$ & $<0.0001$ & $1.4 \%$ & $3.0 \%$ & $<0.0001$ \\
\hline Non-septic severe infection & $5.6 \%$ & $6.9 \%$ & $<0.0001$ & $5.6 \%$ & $6.8 \%$ & $<0.0001$ \\
\hline Solid tumour & $36.6 \%$ & $31.6 \%$ & $<0.0001$ & $37.1 \%$ & $35.6 \%$ & $<0.0001$ \\
\hline Acute leukaemia & $0.6 \%$ & $0.6 \%$ & 0.21 & $0.4 \%$ & $0.7 \%$ & $<0.0001$ \\
\hline
\end{tabular}

*T2DM versus non-DM. $p$ Values were calculated by $t$-test for age and by $\chi^{2}$ test for variables other than age.

T2DM, type 2 diabetes mellitus. 
Table 2 ORs for the risk of DIC in each study population*

\begin{tabular}{|c|c|c|c|c|}
\hline & \multicolumn{2}{|c|}{$\begin{array}{l}\text { Study population A (overall } \\
\text { population) }\end{array}$} & \multicolumn{2}{|c|}{$\begin{array}{l}\text { Study population B (laboratory } \\
\text { data-available admissions only) }\end{array}$} \\
\hline & non-DM & T2DM & non-DM & T2DM \\
\hline \multicolumn{5}{|l|}{ All } \\
\hline Number of admission & 306100 & 260091 & 259633 & 53793 \\
\hline Number of DIC & $3287(1.1 \%)$ & $4640(1.8 \%)$ & $821(0.3 \%)$ & $307(0.6 \%)$ \\
\hline $\begin{array}{l}\text { Age, sex and admission } \\
\text { year-adjusted OR }\end{array}$ & 1 & $1.60(1.52$ to 1.67$)$ & 1 & $1.63(1.43$ to 1.87$)$ \\
\hline $\begin{array}{l}\text { Further adjusting for } \\
\text { underlying diseases }\end{array}$ & 1 & $1.39(1.32$ to 1.45$)$ & 1 & $1.28(1.11$ to 1.47$)$ \\
\hline \multicolumn{5}{|l|}{ By underlying disease } \\
\hline \multicolumn{5}{|l|}{ Sepsis } \\
\hline Number of admission & 5005 & 9211 & 3524 & 1611 \\
\hline Number of DIC & $920(18.4 \%)$ & $1618(17.6 \%)$ & $335(9.5 \%)$ & $125(7.8 \%)$ \\
\hline $\begin{array}{l}\text { Age, sex and admission } \\
\text { year-adjusted OR }\end{array}$ & 1 & 0.97 (0.88 to 1.06$)$ & 1 & 0.79 (0.64 to 0.99$)$ \\
\hline $\begin{array}{l}\text { Further adjusting for } \\
\text { underlying diseases }\end{array}$ & 1 & $0.98(0.90$ to 1.08$)$ & 1 & $0.81(0.65$ to 1.01$)$ \\
\hline \multicolumn{5}{|l|}{ Non-septic severe infection } \\
\hline Number of admission & 17118 & 17931 & 14541 & 3681 \\
\hline Number of DIC & $226(1.3 \%)$ & $527(2.9 \%)$ & $73(0.5 \%)$ & $47(1.3 \%)$ \\
\hline $\begin{array}{l}\text { Age, sex and admission } \\
\text { year-adjusted OR }\end{array}$ & 1 & $2.05(1.75$ to 2.41$)$ & 1 & 2.11 (1.44 to 3.07$)$ \\
\hline $\begin{array}{l}\text { Further adjusting for } \\
\text { underlying diseases }\end{array}$ & 1 & 1.67 (1.41 to 1.97$)$ & 1 & 1.56 (1.05 to 2.30$)$ \\
\hline \multicolumn{5}{|l|}{ Solid tumour } \\
\hline Number of admission & 111908 & 82305 & 96398 & 19127 \\
\hline Number of DIC & $1207(1.1 \%)$ & $1645(2.0 \%)$ & $302(0.3 \%)$ & $115(0.6 \%)$ \\
\hline $\begin{array}{l}\text { Age, sex and admission } \\
\text { year-adjusted OR }\end{array}$ & 1 & 1.89 (1.75 to 2.04$)$ & 1 & $1.93(1.55$ to 2.40$)$ \\
\hline $\begin{array}{l}\text { Further adjusting for } \\
\text { underlying diseases }\end{array}$ & 1 & 1.59 (1.47 to 1.72$)$ & 1 & 1.46 (1.16 to 1.83$)$ \\
\hline \multicolumn{5}{|l|}{ Acute leukaemia } \\
\hline Number of admission & 1809 & 1604 & 1148 & 386 \\
\hline Number of DIC & $451(24.9 \%)$ & $284(17.7 \%)$ & $166(14.5 \%)$ & $33(8.5 \%)$ \\
\hline $\begin{array}{l}\text { Age, sex and admission } \\
\text { year-adjusted OR }\end{array}$ & 1 & $0.73(0.61$ to 0.86$)$ & 1 & $0.57(0.38$ to 0.84$)$ \\
\hline $\begin{array}{l}\text { Further adjusting for } \\
\text { underlying diseases }\end{array}$ & 1 & 0.70 (0.59 to 0.84$)$ & 1 & 0.58 (0.39 to 0.87$)$ \\
\hline
\end{tabular}

Adjustment variables were age (continuous), sex (male or female), admission year (<2011, 2011, 2012, 2013 or 2014) and underlying diseases (sepsis, non-septic severe infection, solid tumour and acute leukaemia; yes or no, for each).

*DIC was defined based on the physician's diagnosis of DIC only in study population A, and the physician's diagnosis of DIC with supporting laboratory data in study population B.

DIC, disseminated intravascular coagulation; T2DM, type 2 diabetes mellitus.

times for those with non-septic severe infection and 1.33 times for those with acute leukaemia higher than that in non-DM group.

Since some antidiabetic medications may modify the association between T2DM and DIC, we performed an analysis stratified by these medications, when given in the 30-day period prior to admission (study population C, comprising 148105 admissions; table 4).

Higher risk of DIC was observed in T2DM patients treated with insulin, relative to those not treated with insulin (fully adjusted OR: 1.53 (1.37 to 1.72)). Recent use of dipeptidyl peptidase-4 (DPP4) inhibitor, $\alpha$-glucosidase inhibitor $(\alpha$-GI) or metformin, however, was significantly associated with a lower risk of DIC.

\section{DISCUSSION}

We found that T2DM was associated with a higher risk of DIC, particularly when it had been treated recently with insulin. When stratified by the diseases underlying DIC, the association between T2DM and DIC was positive for admissions with non-septic severe infection or solid tumour, null among admissions with sepsis and negative among admissions with acute leukaemia.

DM is characterised by a high risk of atherothrombotic disorders. As a result, the association of diabetes with coagulation, thrombosis and fibrinolysis has been studied extensively. Despite this, we were able to find only a dozen case reports on DIC in diabetes patients, and no observational research examining the association 
Table 3 Case fatality of admissions

\begin{tabular}{llll}
\hline & Non-DM & T2DM & Fatality ratio \\
\hline All & $28.9 \%(949 / 3287)$ & $34.2 \%(1586 / 4640)$ & 1.18 \\
Sepsis & $36.0 \%(331 / 920)$ & $39.2 \%(635 / 1618)$ & 1.09 \\
Non-septic severe infection & $28.3 \%(64 / 226)$ & $34.7 \%(183 / 527)$ & 1.23 \\
Solid tumour & $37.5 \%(453 / 1207)$ & $38.4 \%(631 / 1645)$ & 1.02 \\
Acute leukaemia & $25.9 \%(117 / 451)$ & $34.5 \%(98 / 284)$ & 1.33 \\
\hline T2DM, type 2 diabetes mellitus. & & &
\end{tabular}

between diabetes and DIC. Several recent studies of DIC have used the administrative database in Japan, the Japanese Diagnosis Procedure Combination (DPC). The DPC database includes administrative claims and discharge abstract data for all hospitalised patients discharged from more than 1000 hospitals. ${ }^{16-21}$ Most of these studies have examined the association between DIC and the administration of antithrombin or thrombomodulin, but they do not consider the association between DIC and diabetes. Atherothrombotic complications are the main cause of mortality in patients with diabetes. Premature atherosclerosis, increased platelet reactivity and activation of coagulation factors with associated hypofibrinolysis all contribute to increased cardiovascular risk in diabetic patients. ${ }^{8} 9$ Increased plasma levels and/or activity of haemostatic factors, including tissue factor, ${ }^{22}$ Factor VII (FVII), ${ }^{23}{ }^{24}$ thrombin, ${ }^{22}{ }^{25}$ fibrinogen $^{26-28}$ and plasminogen activator inhibitor-1 (PAI-1) ${ }^{29}{ }^{30}$ have all been reported in diabetes patients. The pathogenesis of DIC is similar, in terms of persistent widespread activation of coagulation in the presence of underlying disease.

The degree of fibrinolysis nevertheless varies among the underlying diseases and results in the patient developing three types of clinical manifestation. ${ }^{1}$ First, DIC with suppressed fibrinolysis is usually seen in sepsis. In sepsis, coagulation activation is severe but fibrinolytic activation is mild. This is because PAI is overexpressed in the vascular endothelium as a result of the action of lipopolysaccharide and cytokines. Many microthrombi remain, therefore, and microcirculatory dysfunction leads to the progression of multiple organ failure. ${ }^{3} 31$ Second, DIC with enhanced fibrinolysis is seen in APL. In APL, coagulation and fibrinolytic activation are severe, and fibrinolysis is strongly activated in response to the slightest elevation in PAI. As a result, haemostatic plugs (thrombi due to haemostasis) are more easily dissolved, and the symptoms of severe bleeding appear. However, organ dysfunction seldom occurs. Third, DIC with balanced fibrinolysis is usually seen in solid tumours, with an intermediate pathogenesis between the first two types described above. In this study, a higher risk of DIC was observed in T2DM patients with non-septic severe infection or solid cancer. A potential cause of this higher risk is the suppressed fibrinolysis by increased PAI due to T2DM, which might promote organ failure. Contrary to our expectation, the risk of
DIC was not elevated in T2DM patients with sepsis. One potential explanation is that because fibrinolysis is so suppressed in sepsis that the antifibrinolytic nature of T2DM does not contribute to the development of DIC in patients with sepsis. A lower risk of DIC was observed in T2DM patients with acute leukaemia. This may be the result of the elevated PAI in T2DM suppressing the activated fibrinolysis, which may lead to attenuated bleeding symptoms, resulting in fewer DIC diagnoses than in the non-DM population. In fact, the risk of DIC was even lower in the subgroup including patients with APL only. In T2DM patients, platelets are thought to adhere to the vascular endothelium and aggregate more readily than in healthy people, ${ }^{32}$ which increase the risk of DIC. The contribution of platelets to the development of DIC is thought to be relatively lower in T2DM patients with acute leukaemia because their platelet count is decreased. For study population B, the association with diabetes for each of the diseases underlying DIC was similar to that for study population A. The higher fatality rate in T2DM group presented in table 3 may be due to augmented organ failure caused by increased coagulation and/or suppressed fibrinolysis in T2DM.

In the analysis stratified by type of antidiabetic medication given within the 30-day period prior to admission, T2DM patients treated with insulin had a higher risk of DIC than patients not treated with insulin. Insulin-treated T2DM subjects were reported to be at greater risk of cardiovascular events, which may simply be a reflection of longer disease duration increasing the risk of complications. ${ }^{33}$ Hyperinsulinaemia is associated with prothrombotic changes (increases in fibrinogen and PAI levels) in T2DM patients and community-based cohorts. $^{34} 35$ The higher risk associated with recent insulin use that we observed may thus be the result of increases in fibrinogen and PAI associated with hyperinsulinaemia. Insulin resistance is thought to increase in T2DM patients immediately before hospitalisation, because of the stress of the disease necessitating hospitalisation. Hyperinsulinaemia is therefore likely to develop in such situations, when insulin is administered. However, we cannot identify the cause of the higher risk with certainty, because of a lack of data regarding the duration and severity of diabetes, degree of insulin resistance and the dosage of insulin needed to control blood glucose. The lower risk of DIC observed in recent users of DPP4 inhibitor, $\alpha$-GI or metformin is supported by 
Table 4 Effect of antidiabetic medications* on risk of DIC

\begin{tabular}{|c|c|c|c|c|c|c|c|}
\hline & Insulin & DPP4 inhibitor & su & $\alpha$-GI & Metformin & TZD & Other \\
\hline \multicolumn{8}{|l|}{ All $(n=148105)$} \\
\hline Number of admission & 72583 & 50733 & 40854 & 30359 & 26722 & 14249 & 14429 \\
\hline Number of DIC & 1355 & 507 & 401 & 300 & 228 & 137 & 128 \\
\hline Age, sex and admission year-adjusted OR & 1.88 (1.68 to 2.10$)$ & 0.89 (0.79 to 0.99$)$ & 0.87 (0.77 to 0.98$)$ & 0.83 (0.73 to 0.95$)$ & 0.76 (0.66 to 0.88$)$ & $0.88(0.74$ to 1.05$)$ & $0.71(0.59$ to 0.85$)$ \\
\hline Further adjusting for underlying diseases & $1.53(1.37$ to 1.72$)$ & 0.86 (0.76 to 0.96$)$ & $0.90(0.79$ to 1.01$)$ & 0.85 (0.74 to 0.96$)$ & 0.81 (0.70 to 0.93$)$ & 0.89 (0.74 to 1.07$)$ & 0.74 (0.62 to 0.90$)$ \\
\hline \multicolumn{8}{|c|}{ (1) } \\
\hline \multicolumn{8}{|l|}{ Sepsis $(n=5109)$} \\
\hline Number of admission & 3377 & 1527 & 1002 & 848 & 625 & 329 & 403 \\
\hline Number of DIC & 541 & 173 & 121 & 102 & 75 & 41 & 47 \\
\hline Age, sex and admission year-adjusted OR & 1.33 (1.09 to 1.63$)$ & 0.85 (0.69 to 1.04$)$ & $0.90(0.72$ to 1.14$)$ & $0.89(0.70$ to 1.12$)$ & $0.91(0.70$ to 1.19$)$ & $0.94(0.66$ to 1.33$)$ & $0.84(0.61$ to 1.16$)$ \\
\hline Further adjusting for underlying diseases & 1.34 (1.09 to 1.64$)$ & 0.84 (0.69 to 1.03$)$ & $0.90(0.72$ to 1.13$)$ & $0.89(0.70$ to 1.12$)$ & $0.92(0.70$ to 1.20$)$ & 0.93 (0.65 to 1.31$)$ & 0.85 (0.61 to 1.17$)$ \\
\hline \multicolumn{8}{|l|}{ Non-septic severe infection $(n=10256)$} \\
\hline Number of admission & 5796 & 3105 & 2577 & 2039 & 1554 & 952 & 921 \\
\hline Number of DIC & 175 & 69 & 46 & 39 & 23 & 15 & 17 \\
\hline Age, sex and admission year-adjusted OR & $1.25(0.91$ to 1.71$)$ & $0.99(0.72$ to 1.36$)$ & $0.77(0.54$ to 1.10$)$ & $0.82(0.57$ to 1.18$)$ & $0.62(0.40$ to 0.96$)$ & $0.71(0.41$ to 1.22$)$ & $0.75(0.45$ to 1.24$)$ \\
\hline Further adjusting for underlying diseases & 1.01 (0.73 to 1.40$)$ & 0.91 (0.66 to 1.27$)$ & $0.78(0.54$ to 1.12$)$ & $0.87(0.60$ to 1.25$)$ & $0.64(0.41$ to 1.01$)$ & $0.75(0.43$ to 1.30$)$ & $0.80(0.47$ to 1.33$)$ \\
\hline \multicolumn{8}{|l|}{ Solid tumour ( $n=46590)$} \\
\hline Number of admission & 22702 & 16178 & 13801 & 9078 & 7117 & 4544 & 4288 \\
\hline Number of DIC & 441 & 199 & 164 & 112 & 94 & 54 & 61 \\
\hline Age, sex and admission year-adjusted OR & 1.45 (1.22 to 1.73$)$ & $0.86(0.72$ to 1.03$)$ & $0.81(0.67$ to 0.98$)$ & 0.85 (0.69 to 1.04$)$ & $0.97(0.78$ to 1.21$)$ & $0.86(0.65$ to 1.15$)$ & $0.95(0.73$ to 1.25$)$ \\
\hline Further adjusting for underlying diseases & $1.31(1.09$ to 1.56$)$ & $0.82(0.68$ to 0.98$)$ & $0.88(0.73$ to 1.07$)$ & $0.86(0.70$ to 1.07$)$ & $1.04(0.83$ to 1.30$)$ & $0.88(0.66$ to 1.18$)$ & $1.02(0.77$ to 1.34$)$ \\
\hline \multicolumn{8}{|l|}{ Acute leukaemia $(n=895)$} \\
\hline Number of admission & 528 & 317 & 258 & 173 & 148 & 98 & 58 \\
\hline Number of DIC & 78 & 26 & 23 & 15 & 14 & 9 & 3 \\
\hline Age, sex and admission year-adjusted OR & $1.94(1.17$ to 3.21$)$ & 0.87 (0.50 to 1.50$)$ & $0.84(0.49$ to 1.42$)$ & $0.86(0.47$ to 1.56$)$ & 1.09 (0.57 to 2.09$)$ & 0.76 (0.36 to 1.62$)$ & $0.42(0.12$ to 1.43$)$ \\
\hline Further adjusting for underlying diseases & 1.77 (1.06 to 2.95$)$ & $0.86(0.50$ to 1.50$)$ & $0.82(0.48$ to 1.40$)$ & 0.81 (0.45 to 1.48$)$ & 1.11 (0.58 to 2.13$)$ & $0.70(0.33$ to 1.51$)$ & $0.39(0.11$ to 1.37$)$ \\
\hline
\end{tabular}


previous research. Specifically, reports have shown that DPP4 inhibitor decreases PAI in T2DM patients, ${ }^{36} \alpha$-GI decreases PAI and fibrinogen by improving hyperinsulinaemia, ${ }^{37}$ and that metformin reduces elevated levels of PAI and FVII. ${ }^{38}$

The strengths of the present study include our large sample size and the use of a nationwide claims database. Both of these design elements minimised our sampling bias and the possibility of type II error. In addition, a number of medical institutions also provided laboratory data, which were useful for validating outcomes. The robustness of the findings of the primary and sensitivity analyses suggest that the probability of type I error in these findings is small. Moreover, the generalisability of this study is relatively high. In study population A, 1.8\% (T2DM group) or $1.1 \%$ (non-DM group) of admissions had been diagnosed with DIC, and the case fatality rate for DIC admissions was $34.2 \%$ (T2DM group) or $28.9 \%$ (non-DM group). These DIC diagnosis numbers were comparable to those from the nationwide questionnaire survey conducted by the Japanese Society of Thrombosis Hemostasis/DIC subcommittee in 2009 (in that survey the prevalence of DIC was $1.3 \%$, and the frequency of fatal outcomes was $40 \%){ }^{39}$

In terms of limitations of this study, first, we acknowledge that misdiagnosis of DIC is probably inevitable. In study population $\mathrm{B}$, the frequency of diagnosis of DIC was more than double that with supporting laboratory data $(0.9 \%$ vs $0.4 \%)$. One potential explanation for this difference in frequency is that the DIC diagnoses based on supporting laboratory data only took platelet count and PT/INR into account. It did not include other important parameters such as PR, PT, fibrinogen or FDP, because such data were unavailable. Another potential explanation is that the difference in the case fatality rate $(53.7 \%$ vs $36.3 \%)$ implies that patients with DIC and supporting laboratory data were in a more serious condition than those without laboratory data. Overdiagnosis of DIC probably also contributed to this difference. Although such misclassifications may occur, we found similar associations in both the primary analysis, using the reported DIC cases, and the sensitivity analysis, using the DIC cases defined according to the reported diagnosis of DIC and the supporting laboratory data. FDP/ D-dimer level is especially important for the diagnosis of DIC. Although these values are not available in MDV database whether FDP/D-dimer was measured can be known from the claim data. Of the 1128 admissions with diagnosis of DIC supported by laboratory data in study population B, FDP/D-dimer were measured together with platelet counts and PT in $73.4 \%$ of the admissions (828 admissions). Second, due to the limited access to the MDV data, we cannot include admissions of non-DM patients in the hospitals not providing laboratory data, which may induce selection bias. The impact of the selection bias, however, may be small since the baseline characteristics for admissions of T2DM patients were similar between hospitals providing laboratory data and those not providing laboratory data. Third, the data regarding the use of antidiabetic medication within 30 days prior to admission may not be complete, because data from the primary care clinics and hospitals were not available. Finally, we were unable to eliminate the impact of potential confounding factors such as levels of haemostatic factors, insulin resistance and hydration status, because of the lack of data.

In summary, we found that T2DM is associated with a higher risk of DIC, particularly in patients treated recently with insulin, and among admissions with solid tumour or non-septic severe infection. In clinical practice, physicians should be more suspicious in T2DM patients who present with typical signs and symptoms of DIC, especially in patients treated recently with insulin, and among admissions with solid tumour or non-septic severe infections. However, as this is the first study to show a quantitative association between T2DM and DIC, it would be important to see whether other studies support our study findings.

Contributors $\mathrm{KN}$ and IM designed the study, performed statistical analysis and drafted the manuscript. IM and $\mathrm{H}$ Iso provided statistical expertise. All authors contributed to the interpretation of the data and the critical revision of the manuscript and approved the final version. $\mathrm{KN}, \mathrm{IM}$ and $\mathrm{H}$ Iso are the guarantors of the current investigation.

Funding This study was conducted using the data from MDV purchased by the Takeda Pharmaceutical Company by which the lead author, KN, was employed.

Competing interests KN received salary from the Takeda Pharmaceutical Company.

Ethics approval The present study was approved by the Ethics Committee of the Graduate School of Medicine/Faculty of Medicine, Osaka University (approval number 16090).

Provenance and peer review Not commissioned; externally peer reviewed.

Data sharing statement No additional data are available.

Transparency declaration The lead author affirms that the manuscript is an honest, accurate and transparent account of the study being reported; that no important aspects of the study have been omitted and that any discrepancies from the study as planned have been explained.

Open Access This is an Open Access article distributed in accordance with the Creative Commons Attribution Non Commercial (CC BY-NC 4.0) license, which permits others to distribute, remix, adapt, build upon this work noncommercially, and license their derivative works on different terms, provided the original work is properly cited and the use is non-commercial. See: http:// creativecommons.org/licenses/by-nc/4.0/

\section{REFERENCES}

1. Asakura $\mathrm{H}$. Classifying types of disseminated intravascular coagulation: clinical and animal models. J Intensive Care 2014;2:20.

2. Levi M, van der Poll T. Disseminated intravascular coagulation: a review for the internist. Intern Emerg Med 2013;8:23-32.

3. Levi M, Ten Cate H. Disseminated intravascular coagulation. $N$ Eng J Med 1999;341:586-92.

4. Wada $\mathrm{H}$, Asakura $\mathrm{H}$, Okamoto $\mathrm{K}$, et al. Japanese Society of Thrombosis Hemostasis/DIC Subcommittee. Expert consensus for the treatment of disseminated intravascular coagulation in Japan. Thromb Res 2010;125:6-11.

5. Nakagawa M. An investigation report concerning incidence and underlying cause of DIC in Japan: a study report by the research committee on blood coagulation on abnormalities in 1998. Special diseases designated by Japanese Ministry of Health and Welfare, 1999:157-64. Japanese. 
6. Dhainaut JF, Yan SB, Joyce DE, et al. Treatment effects of drotrecogin alfa (activated) in patients with severe sepsis with or without overt disseminated intravascular coagulation. $J$ Thromb Haemost 2004;2:1924-33.

7. Ogura H, Gando S, Saitoh D, et al. Japanese Association for Acute Medicine Sepsis Registry (JAAMSR) Study Group. Epidemiology of severe sepsis in Japanese intensive care units: a prospective multicenter study. $J$ Infect Chemother 2014;20:157-62.

8. Alzahrani SH, Ajjan RA. Coagulation and fibrinolysis in diabetes. Diab Vasc Dis Res 2010;7:260-73.

9. Grant PJ. Diabetes mellitus as a prothrombotic condition. $J$ Intern Med 2007:262:157-72.

10. Giovannucci E, Harlan DM, Archer MC, et al. Diabetes and cancer: a consensus report. Diabetes Care 2010;33:1674-85.

11. Hanatani T, Sai K, Tohkin M, et al. Impact of Japanese regulatory action on metformin-associated lactic acidosis in type II diabetes patients. Int J Clin Pharm 2015;37:537-45.

12. Hori K, Kobayashi $\mathrm{N}$, Atsumi $\mathrm{H}$, et al. Changes in compliance with Japanese antiemetic guideline for chemotherapy-induced nausea and vomiting: a nationwide survey using a distributed research network. Support Care Cancer 2014;22:969-77.

13. Ueyama $\mathrm{H}$, Hinotsu $\mathrm{S}$, Tanaka $\mathrm{S}$, et al. Application of a self-controlled case series study to a database study in children. Drug Saf 2014;37:259-68.

14. Chang $\mathrm{CH}$, Kusama M, Ono S, et al. Assessment of statin-associated muscle toxicity in Japan: a cohort study conducted using claims database and laboratory information. BMJ Open 2013;3:e002040.

15. Urushihara $\mathrm{H}$, Taketsuna M, Liu Y, et al. Increased risk of acute pancreatitis in patients with type 2 diabetes: an observational study using a Japanese hospital database. PLoS One 2012;7:e53224.

16. Murata A, Okamoto K, Mayumi T, et al. Observational study to compare antithrombin and thrombomodulin for disseminated intravascular coagulation. Int J Clin Pharm 2015;37:139-47.

17. Murata A, Okamoto K, Mayumi T, et al. The recent time trend of outcomes of disseminated intravascular coagulation in Japan: an observational study based on a national administrative database. $J$ Thromb Thrombolysis 2014;38:364-71.

18. Tagami T, Matsui $\mathrm{H}$, Horiguchi $\mathrm{H}$, et al. Antithrombin and mortality in severe pneumonia patients with sepsis-associated disseminated intravascular coagulation: an observational nationwide study. $J$ Thromb Haemost 2014;12:1470-9.

19. Tagami T, Matsui H, Fushimi K, et al. Use of recombinant human soluble thrombomodulin in patients with sepsis-induced disseminated intravascular coagulation after intestinal perforation. Front Med 2015;2:7.

20. Tagami T, Matsui $\mathrm{H}$, Horiguchi $\mathrm{H}$, et al. Recombinant human soluble thrombomodulin and mortality in severe pneumonia patients with sepsis-associated disseminated intravascular coagulation: an observational nationwide study. J Thromb Haemost 2015:13:31-40.

21. Tagami T, Matsui H, Fushimi K, et al. Supplemental dose of antithrombin use in disseminated intravascular coagulation patients after abdominal sepsis. Thromb Haemost 2015;114:537-45.

22. Boden G, Vaidyula VR, Homko C, et al. Circulating tissue factor procoagulant activity and thrombin generation in patients with type 2 diabetes: effects of insulin and glucose. $J$ Clin Endocrinol Metab 2007:92:4352-8.

23. Heywood DM, Mansfield MW, Grant PJ. Factor VII gene polymorphisms, factor VII:C levels and features of insulin resistance in non-insulin-dependent diabetes mellitus. Thromb Haemost 1996;75:401-6.

24. Karatela RA, Sainani GS. Interrelationship between coagulation factor VII and obesity in diabetes mellitus (type 2). Diabetes Res Clin Pract 2009;84:e41-4.

25. Undas A, Wiek I, Stepien E, et al. Hyperglycemia is associated with enhanced thrombin formation, platelet activation, and fibrin clot resistance to lysis in patients with acute coronary syndrome. Diabetes Care 2008;31:1590-5.

26. Klein RL, Hunter SJ, Jenkins AJ, et al., The DCCT/EDIC Study Group. Fibrinogen is a marker for nephropathy and peripheral vascular disease in type 1 diabetes: studies of plasma fibrinogen and fibrinogen gene polymorphism in the DCCT/EDIC cohort. Diabetes Care 2003;26:1439-48.

27. Barazzoni R, Kiwanuka E, Zanetti M, et al. Insulin acutely increases fibrinogen production in individuals with type 2 diabetes but not in individuals without diabetes. Diabetes 2003;52:1851-6.

28. Tessari $\mathrm{P}$, Kiwanuka $\mathrm{E}$, Millioni R, et al. Albumin and fibrinogen synthesis and insulin effect in type 2 diabetic patients with normoalbuminuria. Diabetes Care 2006;29:323-8.

29. Seljeflot I, Larsen JR, Dahl-Jorgensen K, et al. Fibrinolytic activity is highly influenced by long-term glycemic control in type 1 diabetic patients. J Thromb Haemost 2006;4:686-8.

30. Juhan-Vague I, Roul C, Alessi MC, et al. Increased plasminogen activator inhibitor activity in noninsulin dependent diabetic patientsrelationship with plasma insulin. Thromb Haemost 1989;61:370-3.

31. Gando S. Microvascular thrombosis and multiple organ dysfunction syndrome. Crit Care Med 2010;38:S35-42.

32. Vinik AI, Erbas T, Park TS, et al. Platelet dysfunction in type 2 diabetes. Diabetes Care 2001:24:1476-85.

33. Margolis DJ, Hoffstad O, Strom BL. Association between serious ischemic cardiac outcomes and medications used to treat diabetes. Pharmacoepidemiol Drug Saf 2008;17:753-9.

34. Meigs JB, Mittleman MA, Nathan DM, et al. Hyperinsulinemia, hyperglycemia and impaired hemostasis: the Framingham Offspring Study. JAMA 2000;283:221-8.

35. Festa A, D'Agostino R, Mykkanen L, et al. Relative contribution of insulin and its precursors to fibrinogen and PAl-1 in a large population with different states of glucose tolerance. The Insulin Resistance Atherosclerosis Study (IRAS). Arterioscler Thromb Vasc Biol 1999;19:562-8.

36. Tani S, Takahashi A, Nagao K, et al. Effect of dipeptidyl peptidase-4 inhibitor, vildagliptin on plasminogen activator inhibitor-1 in patients with diabetes mellitus. Am J Cardiol 2015;115:454-60.

37. Shinoda Y, Inoue I, Nakano T, et al. Acarbose improves fibrinolytic activity in patients with impaired glucose tolerance. Metab Clin Exp 2006;55:935-9.

38. Grant PJ. Beneficial effects of metformin on haemostasis and vascular function in man. Diabetes Metab 2003;29:6S44-6S52.

39. Morishita E, Asakura H. Recombinant thrombomodulin-hematologic malignancy. J Clin Exp Med 2011;283:107-13. Japanese. 